Questions vives

\section{Questions Vives}

Recherches en éducation

$n^{\circ} 21 \mid 2014$

Le travail collectif des enseignants en question(s)

\title{
De la réification à l'autonomie, les difficultés à penser sociologiquement le travail collectif des enseignants
}

From reification to autonomy, the difficulties to think sociologically the teachers' collective work

\section{Nicolas Sembel}

\section{CpenEdition}

\section{Journals}

Édition électronique

URL : http://journals.openedition.org/questionsvives/1525

DOI : 10.4000/questionsvives.1525

ISSN : $1775-433 \mathrm{X}$

\section{Éditeur}

Université Aix-Marseille (AMU)

Édition imprimée

Date de publication : 15 septembre 2014

ISBN : 978-2-912643-45-2

ISSN : 1635-4079

Référence électronique

Nicolas Sembel, « De la réification à l'autonomie, les difficultés à penser sociologiquement le travail collectif des enseignants », Questions Vives [En ligne], n² 21 | 2014, mis en ligne le 15 septembre 2014, consulté le 03 mai 2019. URL : http://journals.openedition.org/questionsvives/1525; DOI : 10.4000/ questionsvives.1525

Ce document a été généré automatiquement le 3 mai 2019.

\section{(†)}

Questions Vives est mis à disposition selon les termes de la licence Creative Commons Attribution Pas d'Utilisation Commerciale - Pas de Modification 4.0 International. 


\section{De la réification à l'autonomie, les difficultés à penser sociologiquement le travail collectif des enseignants} From reification to autonomy, the difficulties to think sociologically the teachers'
collective work

Nicolas Sembel

\section{Introduction}

1 Des ouvrages d'acteurs politiques ou syndicaux de premier plan peuvent parfois être des sources d'inspiration, ou mieux, des bases de travail, pour les chercheurs. Celui du syndicaliste italien Bruno Trentin (1926-2007), intitulé La cité du travail. Le fordisme et la gauche (1997/2012), introduit par Alain Supiot, professeur au Collège de France, fait le point sur l'incapacité théorique à penser le travail, qui entraîne une incapacité politique (et syndicale) à résister à l'offensive idéologique libérale sur ce terrain. Dans un autre registre, scientifique, qui présente une analogie avec le précédent, il existe une certaine incapacité sociologique à penser le travail collectif des enseignants. En sociologie du travail, cet objet se situe tout en bas de la hiérarchie, comme le travail enseignant en général, laissé à la sociologie de l'éducation. De son côté, cette dernière dénie souvent au travail collectif des enseignants jusqu'à sa dimension empirique: défini strictement comme un travail d'équipe, il n'existerait pas dans un milieu réputé individualiste (Dubet, 1991; Dubet \& Martuccelli, 1996; Barrère, 2002), individualisme qui reste toutefois à questionner (Geay, 2010). Le premier problème est celui de la définition du phénomène : toujours d'un point de vue sociologique, une telle définition ne peut être que large, au moins dans un premier temps; à la dimension formelle de travail d'équipe, que nous venons d'évoquer, s'ajoute une dimension informelle. 
2 Sur la base de cette acception large, nous avons constaté, dans nos recherches, l'existence d'un travail collectif enseignant important sur le plan informel. Sans surprise, l'informel prédomine, car décrire «ce qui se passe » réellement est ce qui intéresse le plus la sociologie du travail. De ce point de vue, le travail d'équipe formalisé n'est qu'une variante du travail collectif, et pas forcément la plus intéressante, ni la plus répandue. Les deux dimensions, formelle et informelle, peuvent être regroupées dans ce que Woods (1990) appelle la dimension relationnelle du travail. Cet auteur y inclut aussi la relation pédagogique aux élèves : objectivement, un enseignant n'est jamais vraiment seul. Il faudrait en toute rigueur également ajouter un dernier type de «rencontre» professionnelle propre au travail intellectuel : les «discussions » avec les auteurs morts ou vivants, lus et jamais rencontrés "physiquement», qui influencent tant le travail enseignant et participent pleinement, selon nous, de la construction « intellectuelle » de sa dimension relationnelle, si ce n'est collective, des prises de position du travailleur dans le groupe ou tout autre collectif, de sa motivation, souvent intrinsèque, à travailler, etc. Dans cette perspective, chaque bibliographie est la mise en forme d'un "réseau cartographique » intellectuel et, finalement, l'expression d'un travail collectif.

Pour définir le travail collectif des enseignants par sa dimension empirique, nous nous appuierons sur deux contextes professionnels et un terrain d'enquête qui permettent de l'aborder sous plusieurs angles complémentaires. Nous ne chercherons pas à transformer cette notion, et les notions voisines qui servent à l'illustrer, en concepts (cf. Marcel, 2006, pour une approche détaillée théoriquement et validée empiriquement); mais seulement à questionner le travail enseignant par sa dimension collective, d'abord par ses facettes empiriques.

4 Une fois établie sociologiquement, comme nous venons de le décrire dans cette introduction, l'existence empirique du travail collectif enseignant, et indiqué quelques pistes possibles pour sa théorisation, bref avoir entamé sa construction en objet d'étude après avoir fait la preuve de sa visibilité, il reste à questionner sa centralité, notamment théorique (partie 1), professionnelle (partie 2, 2.1 à 2.3.), sociologique (2.4.), et à en discuter les principaux résultats obtenus (partie 3). Un tel objet, qui reste marginal, éclaire-t-il les "grandes » questions qui traversent la thématique du travail? Nous voudrions défendre dans cet article une réponse positive à cette question; ce qui est aussi une manière de lier les difficultés à penser collectivement le travail enseignant aux difficultés à penser le travail enseignant tout court.

\section{Cadre théorique}

Plusieurs éléments théoriques contextualisent le travail enseignant collectif aujourd'hui, traduisant des transformations structurelles; pour aller directement au cœur de notre propos, nous mettrons l'accent sur sept d'entre eux, sans développer autant qu'il le faudrait leur dimension historique et leurs caractéristiques théoriques et sociales propres. Notre objectif est, au contraire, de faire ressortir un lien qui existe entre eux, un lien qui ne détaille pas les nuances du travail collectif des enseignants mais qui le replace dans des enjeux sociétaux. Le travail collectif des enseignants relève d'abord de la sociabilité professionnelle, concept descriptif de base (1.1.). Nous le déclinerons ensuite autour de trois «couples»: compétence individuelle/solidarité collective (1.2.), qualification/déqualification (1.3.), réification/intellectualisation (1.4.). Le travail 
enseignant est, comme tout phénomène social, un enjeu de luttes, comme nous le verrons à travers les trois exemples présentés en partie 2 .

\subsection{Sociabilité professionnelle}

6 Nous aborderons la vaste question de la sociabilité professionnelle à partir de la problématique de la force des liens faibles de Granovetter (1973/2008), et de son opérationnalisation par la variation autour des deux dimensions de la fréquence et de l'intensité des « rencontres ». Celle-ci est bien connue et souligne l'importance des « liens faibles ", à faible intensité, et parfois faible fréquence, mais dont la «force" s'avère souvent décisive dans les relations humaines, en l'occurrence l'obtention d'un emploi dans l'exemple retenu par Granovetter. Par extension, des travaux français sur la sociabilité en général ont fait varier des «modèles» de relations humaines diverses (familiales, amicales, de voisinage et, donc, professionnelles) autour des deux variables de la fréquence et de l'intensité. Fréquence faible ou forte et intensité faible ou forte permettent de décrire quatre cas de figure. Nous appliquerons cette grille de lecture au travail collectif des enseignants, à travers l'exploitation de nos données d'enquête (cf. 2.4.). Les liens faibles correspondent aux rencontres à faible intensité, voire à faible fréquence, ce qui donne une consistance empirique à un travail «informel», comme forme la plus « simple », à ne pas négliger, du travail collectif des enseignants. Il y a aussi les " liens forts ", et un tel travail collectif peut aussi être intense, fréquent et formalisé, autant autour d'un travail de recherche, de recherche-action, de recherche documentaire, que de mise en place ou de régulation d'un enseignement. Ce qu'il importe de retenir en introduction, c'est la diversité des formes réelles ou potentielles du phénomène, dans laquelle il faut aussi inclure les relations avec des collègues en poste dans d'autres écoles ou établissements (collègues de promotion d'IUFM, anciens collègues).

\subsection{Compétence individuelle versus solidarité collective}

7 Point commun de ces collectifs de nature différente, le terme de compétence est aujourd'hui le plus répandu (socle commun des compétences pour les élèves, objectif de maitrise des compétences pour les futurs enseignants, définition de la formation et du métier autour de ces compétences), et le plus ambigu, tant sa dimension collective est complexe à mettre en œuvre. Sans une critique, notamment sociologique, la plus précise possible, sa "réappropriation professionnelle » par le travailleur pour construire une autonomie véritable est très difficile (Sembel, 2013a). La compétence omniprésente peut réifier le travail des enseignants (et des élèves) par sa simplification (Sembel, 2003) et par l'adaptation permanente qu'elle requiert. La dimension politique du contexte actuel est directement reliée à cette alternative théorique autonomie/adaptation.

L'articulation des dimensions formelles et informelles du travail collectif ouvre un espace pour la notion de coopération et le concept de "travail altruiste», proches des problématiques de Durkheim et de Mauss (Sembel \& Gesson, 2011). Leur formulation éclaire le travail collectif des enseignants sous l'angle de la notion inusitée de solidarité professionnelle. Celle-ci actualise l'alternative heuristique précisée en leur temps par Durkheim et Mauss, entre le travailleur " courbé sur sa tâche ", ou au contraire sortant de soi. Les deux sont pris dans le travail altruiste, qui «force l'homme à compter avec 
autrui » (Durkheim, 1893/1986, 394) ; mais le second y est nettement moins forcé que le premier, il s'engage dans le collectif avec plus de détermination; sa coopération au travail est caractérisée par la " dette solidaire » et non par l'échange utilitaire (Sembel \& Gesson, 2011). Il est possible de montrer que l'absence de solidarité professionnelle, au sens de Durkheim et de Mauss, domine le travail collectif des enseignants, et leur travail en général, tels qu'ils sont valorisés par les politiques publiques actuelles. Et la tendance la plus visible est celle qui met en avant la notion de compétence individuelle. Des tendances moins visibles, que nous allons aborder en suivant, sont la déqualification et la réification du travail; contre la dé-intellectualisation qui s'en suit, une réintellectualisation semble être la principale réponse possible. Celle-ci passe, conformément à notre problématique théorique, et à nos données empiriques, par un rapport individuel au travail marqué par l'autonomie et non par l'adaptation. Aujourd'hui, la forme du travail collectif des enseignants est valorisée au détriment du sens qu'il peut avoir. Contre cette tendance, que nous critiquerons, nous montrerons, à l'appui de nos données, que, d'un point de vue sociologique, le sens du travail collectif dépend du rapport individuel au travail entretenu par les enseignants participants aux collectifs de travail (en équipe, en réunion, en binôme, etc.). Les "sortants de soi" donnent aux collectifs de travail un tout autre sens que les « centrés sur soi ».

\subsection{Qualification versus déqualification}

9 Des mécanismes parfois subtils de déqualification (terme qui a quasiment disparu des traités de sociologie du travail et des programmes de recherche) et de qualification (nouvelle) du travail enseignant se croisent. Par exemple, la requalification des instituteurs en professeurs des écoles en France à partir de 1991 a été caractérisée par l'augmentation du niveau scolaire des enseignants du premier degré, et s'est accompagnée de plusieurs processus: un recul de la polyvalence disciplinaire, une redéfinition des missions des enseignants, une délégation de tâches à d'autres collègues spécialisés dans d'autres matières, une sous-traitance d'autres tâches à des personnels de statuts hors Éducation nationale, une spécialisation statutaire possible (toujours à l'ordre du jour) de personnels de direction... L'augmentation de la qualification s'accompagne aussi, dans ce cas, d'un morcellement du travail et d'un renforcement des procédures de contrôle (évaluation et, à terme, hiérarchisation). Par contre, en maternelle, dans le secondaire et dans le supérieur, se mettent en place des processus de déspécialisation déqualifiante : interrogation sur l'utilité de la mastérisation en maternelle, promotion à terme de la bivalence disciplinaire au collège, et généralisation des activités d'enseignement dans le supérieur, au détriment de la recherche, pour les personnels concernés (Sembel, Gesson, Léonard \& Teruel, 2012).

La déqualification se constate également à l'échelle européenne : il existe un processus généralisé de fragmentation interne du travail, d'externalisation des tâches et de valorisation des standards qui émiettent le travail (pour reprendre le terme de Friedmann) et produisent un "trouble enseignant généralisé » (Malet, 2008; Normand, 2007), du fait de l'éloignement du travail enseignant de sa dimension culturelle, notamment par la promotion de standards d'une culture minimale ("SMIC culturel», "Socle commun ", etc). Le travail enseignant individuel et collectif se voit ainsi déqualifié par l'affaiblissement de l'élément qui lui donne le plus de sens : la culture, qui garantit sa 
dimension intellectuelle et universaliste. La réification est au bout du processus de déqualification.

\subsection{Réification versus intellectualisation}

11 Cette tendance à la réification (au sens "actualisé » de Supiot dans l'introduction à la traduction de l'ouvrage de Trentin, 1997/2012) du travail enseignant domine donc même dans les cas de qualification nouvelle, alors qu'elle aurait $\mathrm{pu}$ y être au contraire amoindrie. La question de l'évaluation joue notamment un rôle important dans cette réification. Supiot met l'accent sur le dispositif emblématique de l'entretien d'évaluation qui, sous couvert de «sens» donné au travail, recherche en fait la «mobilisation maximale» du travailleur (selon l'expression, note Supiot, de Carl Schmitt, un des théoriciens de l'État nazi - ce qui parachève ainsi le lien « wébérien » existant selon lui, et selon Trentin, entre taylorisme et fordisme d'une part, capitalisme, soviétisme et nazisme d'autre part) ; dans une logique de "réactivité sans limites » et sans « action » véritable de ce même travailleur. Tout dispositif d'évaluation, de tout travail, contient potentiellement de telles dérives, ce potentiel étant à "fleur de peau », selon l'expression de Supiot. Le problème principal devient alors le frein à l'expression d'un tel potentiel morbide, et non, comme on le croit souvent, la simple prévention des dérives de cette expression. Ces dérives ne dépendent pas de contextes favorables, car le contexte leur est toujours potentiellement favorable; favorisées par n'importe quel contexte, elles ne peuvent être contrées sans freins puissants, qui vont jusqu'à la suppression de toute évaluation qui ne serait pas formative, pas profitable au travailleur (élève, enseignant, autres) évalué. Et, si la découverte des freins les plus puissants possibles est nécessaire, elle même risque de ne pas être suffisante, sans une réflexion critique sur le cadre, souvent inconscient et euphémisé, comme le souligne Bourdieu (1984), notamment à partir du «cas » de l'enseignant Heidegger (Bourdieu, 1988), dans lequel l'évaluation se met en place, au travail et ailleurs.

12 Conformément à l'analyse de Trentin, les collectifs de travail enseignant politiquement situés à gauche ont du mal à se positionner face à cette réification, et certains de ces collectifs la défendent paradoxalement. Ne pas voir le travail enseignant comme un travail «classique», croire que ce travail est dominé par un individualisme mal défini (mais idéologiquement très précis) et que, par conséquent, sa dimension collective doit être conquise contre cette pente individualiste qui serait naturelle, évaluer des compétences en ne se souciant que de la définition de ces termes sans les resituer dans le cadre impensé qui les sous-tend, abandonner toute construction d'un rapport fort à une discipline, toute passion pour sa discipline... Autant d'aspects, dans le domaine du travail enseignant, de la « débâcle de la pensée critique sur la question du travail » (Supiot) et des concessions faites par la gauche à la «variante intellectuelle » du compromis fordiste (ce que les positions de Gramsci annonçaient déjà). La valorisation de l'adaptation individuelle et collective au travail est probablement la concession la plus préjudiciable. Seule une (ré)intellectualisation permanente $d u$ travail enseignant, d'un travail enseignant autonome, notamment par toutes les variantes de sa dimension collective que nous avons énumérées, ferait contrepoids à cette débâcle et écarterait ces concessions.

Les collectifs de travail (au sens «mathématique » de travail à deux, ou plus) n'ont donc pas disparu, ils se divisent entre collectifs de promotion consciente ou inconsciente de cette réification (avec l'utilisation spécifique de termes comme compétence, évaluation, 
adaptation, individualisation, autonomie, etc.) et collectifs de lutte, là encore conscients ou inconscients, contre cette réification.

\section{Deux contextes professionnels et un terrain sociologique}

Le terrain sociologique auquel nous avons fait référence depuis le début ne prend sens que par rapport à un contexte politique que nous avons également évoqué à plusieurs reprises. Deux contextes professionnels et un terrain d'enquête serviront de support pour illustrer les sept éléments théoriques qui viennent d'être présentés. Nous préciserons d'emblée la posture d' « objectivation participante » qui est la nôtre.

\subsection{Une posture d'objectivation participante}

15 Aborder des contextes professionnels vécus de l'intérieur ne va pas de soi ; les aborder de façon critique encore moins. Rien de théorique, ni même de méthodologique, dans ce qui va suivre; mon but est simplement de préciser «d'où je parle». Il ne s'agit donc pas d'observation participante à la manière de l'École de Chicago : il n'y a ni protocole de recherche planifié ni immersion en tant que chercheur dans le milieu étudié. Il s'agit de témoignages de " vécu ", mais d'un vécu de sociologue. Enseignant-chercheur en sociologie , j'ai une inclinaison, propre à la plupart des sociologues, à analyser le plus sociologiquement possible les situations dans lesquelles je suis impliqué, dans lesquelles je participe, j'observe et j'objective. Ceci suppose une certaine distance avec la situation elle-même, donc avec ma position non spécifiquement sociologique dans la situation. Cette distanciation ne peut pas être décrétée ex nihilo, ne peut pas être le fruit d'une simple volonté individuelle; elle doit être reconnue et actée par un statut et un positionnement professionnels, même implicites, et surtout, non univoques. En effet, la multiplicité des points de vue, regroupés en un seul regard de sociologue, est nécessaire. Multiplicité qui comporte un risque, celui du porte-à-faux, mais qui présente un avantage, celui de créer un décalage, lequel peut prendre des formes très diverses et variables. Bref, un sociologue est toujours un sociologue, même hors des situations de recherche planifiée.

Nous avons essayé de construire ce décalage (au sens de: poser d'emblée et cultiver régulièrement) au cœur des deux contextes qui serviront de support à notre argumentation. Notre entreprise est aussi ambitieuse et risquée que nécessaire. S'il est plus difficile, selon nous, pour un sociologue de refouler sa sociologie plutôt que de la laisser s'exprimer, naturellement en un sens, sur les situations qu'il vit, la multiplicité des points de vue se pratique au risque de la confusion des genres et, plus fondamentalement, l'observation participante qui la sous-tend ne peut être affirmée publiquement, elle doit rester cachée aux yeux de la plupart des acteurs (y compris d'autres sociologues) observés à leur insu dans les situations choisies par le sociologue. S'il ne peut pas être militant, il ne peut pas non plus n'être qu'un pur chercheur tout le temps. Et, s'il ne peut pas être neutre, il doit travailler constamment à la neutralisation, qui peut être très variable, de ses prises de position.

Le premier contexte est la mise en place, au niveau local (IUFM d'Aquitaine, aujourd'hui devenu ESPÉ) de la mastérisation de la formation des enseignants. Précisons que nous 
travaillons à l'IUFM/ESPÉ depuis 1997. Nous avons multiplié les prises de responsabilités, les fonctions électives, diverses missions confiées par la direction, les contacts informels, une enquête sociologique sur le travail administratif, etc. Avec la mastérisation, notre positionnement a été immédiatement «critique-constructif». Persuadés que cette réforme était essentiellement un " coup " politique en partie contraint, en partie voulu, nous avons tenté le plus systématiquement possible de faire basculer le rapport de force instauré par ce coup en faveur des intérêts de nos disciplines de sciences sociales. Concrètement: plus de cours et de travaux dirigés de sociologie notamment, plus de transversalité propice à la sociologie dans les formations et auprès des publics, plus d'investissement dans le mémoire (qui n'est plus professionnel, sans être de recherche), pour développer tous azimuts une conception critique de la professionnalisation des enseignants.

Le second contexte, le Conseil national des universités (CNU) de sociologie (section 19), de par la "crise des autopromotions" qu'il a connu, s'est ouvert lors des précédentes élections de 2011 à une liste intersyndicale et inter-associative qui l'a largement emporté. Ainsi, au titre de membre d'une association nationale de sociologues (l'Association des sociologues enseignants du supérieur, l'ASES), j'ai été élu tête de liste suppléant du collège des maîtres de conférences. Je précise que l'ASES n'est ni un syndicat ni une société savante, mais un collectif où, selon moi, le sociologue fait de la politique en tant que sociologue et non en tant que militant. J'ai mis en œuvre cette posture "d'intervention dans le monde social qui obéisse, autant que possible, aux règles en vigueur dans le champ scientifique ", comme le défendait Pierre Bourdieu, tout au long de mon implication à l'ASES (où je suis adhérent depuis 1997 et administrateur depuis 2009), aussi bien à travers la construction de nombreuses prises de positions collectives qu'au sein du CNU. Ni syndiqué ni titulaire, j'ai pu «observer en participant » à une session $\mathrm{CNU}$, et en étant inscrit sur une liste syndicale de diffusion d'informations (celle du Syndicat National de l'Enseignement SUPérieur, le SNESUP, participant à la liste victorieuse, aux côtés de l'ASES, de l'AFS et du SGEN), en plus des listes des élus du CNU (liste officielle, liste restreinte à nos élus).

19 Au final, dans les deux contextes, ce qui peut apparaître pour un individualisme sociologique, peut également, du fait du parti-pris critique, participer du développement de la dimension collective du travail enseignant pris initialement pour objet d'étude (sociologique) et traité ensuite comme problème (social) à résoudre. C'est ce que le troisième exemple, seule "véritable" recherche de terrain, nous permettra de parachever. Notre posture sera alors "pleinement» sociologique, et toujours d'objectivation participante, la dimension socio-analytique des mêmes questions posées sur plusieurs années agissant sur les interviewés, mais aussi sur l'interviewer, travailleur intellectuel également, et confronté aux mêmes réalités.

\subsection{Contexte 1 : la mastérisation des enseignants}

Depuis son «lancement» en janvier 2008 et sa mise en place en septembre 2008, la mastérisation de la formation des futurs enseignants en France n'est pas caractérisée par une revendication, et encore moins une mobilisation, autour de la question de la mastérisation des formateurs, qui n'ont pas le niveau des étudiants qu'ils doivent former. Depuis les contestations "nationales" de la mastérisation à sa mise en place «locale ", jamais la question du travail des formateurs en master non titulaires d'un master, n'a été 
soulevée, à notre connaissance. Elle est pourtant essentielle dans une perspective de qualification et de dé-réification.

Lorsque la question, relevant de la formation continue, de la mastérisation des formateurs non mastérisés a été posée, à titre individuel, par un de nos collègues sans master de l'IUFM d'Aquitaine qui a bien voulu nous rapporter ce fait, dès la rentrée 2008, au service des ressources humaines de l'université de tutelle, il lui a été opposé par téléphone une fin de non-recevoir, la question ne devant pas se poser. Le cas de figure n'avait visiblement jamais été envisagé, ni au niveau « inférieur » de l'IUFM, ni au niveau "supérieur » de la tutelle. Depuis, une formation spécifique a été localement mise en place, à laquelle j'ai participé en tant que formateur en sociologie ; mais j'ai pu constater plusieurs problèmes. On peut dénombrer: l'absence de délégation de temps de formation; la sélectivité excessive, du fait du manque de places proposées par rapport aux besoins ; le népotisme, par le renforcement de réseaux, la priorité étant donnée à des connaissances diverses (par exemple, sur la première promotion d'une petite dizaine d'étudiants-collègues, deux d'entre eux étaient élus au Conseil d'administration d'une mutuelle proche du milieu de l'Éducation nationale, induisant ainsi une surreprésentation spectaculaire de cette population par ailleurs infime); l'absence de campagne d'incitation à suivre cette formation; des cours systématiquement filmés ; une validation parfois « à la baisse » du travail fourni ; etc.

Cette quasi-absence d'offre rejoint l'absence de demande collective, syndicale ou institutionnelle. Les investissements des acteurs collectifs dans la mastérisation sont pourtant considérables, autant sur le plan de la critique que de la mise en place du dispositif, qui tourne parfois à la cogestion entre syndicats et institution. Même les étudiants (usagers et représentants) ne semblent pas s'émouvoir du fait d'être formés à un diplôme par des formateurs qui n'en possèdent ni le niveau ni le titre. La "professionnalisation" ainsi définie se révèle être une formidable entreprise de déqualification $\mathrm{du}$ travail enseignant (le problème ne se posait pas avant la mastérisation). La valorisation de compétences professionnelles sans possession du diplôme auquel on forme constitue une confirmation de la critique de Trentin: ce sont bien des intellectuels professionnels qui participent eux-mêmes de la déqualification, de la réification et de la désintellectualisation de leur travail.

Notre observation participante, basée sur la présence à des réunions et sur des discussions informelles, en discussion avec des acteurs de plusieurs points d'observation (institutionnels, syndicaux, professionnels, estudiantins), nous permet de voir ce que l'indigène ne voit plus; en l'occurrence, cette absence de revendication de mastérisation de formateurs illustre l'espace restreint des possibles des manières de penser, et de ne pas penser, le travail enseignant ; et des conditions et limites pour transformer cette pensée en collectif, que ce soit en action collective ou en collectifs formels de travail. L'exemple suivant illustre $a$ contrario une telle action collective.

\subsection{Contexte 2 : le $\mathrm{CNU} 19$}

24 En mars 2012, la CP-CNU (Conférence permanente du Conseil national des universités) s'est divisée sur la question de l'évaluation des enseignants-chercheurs, dans une période pré-électorale dite de conjoncture «fluide » (Dobry, 1986) ; à cette occasion, l'expression d'une position pourtant critique de la section CNU 19 ne s'est pas accompagnée, au sein de cette section, d'une définition dé-réifiante du travail des enseignants-chercheurs. 

précipitation une "réforme » sur l'évaluation des enseignants-chercheurs, dans la continuité de la loi dite LRU d'août 2007. Les sections CNU sont mobilisées et s'expriment toutes ou presque sur la question. À l'issue du temps de concertation, la CP-CNU, regroupant des membres des bureaux de chaque section, où le syndicat majoritaire est le SNESUP, se prononce sur deux textes, les deux détaillant des modalités et des garde-fous d'une éventuelle future procédure d'évaluation, le texte 1 critiquant (tout en l'acceptant) cette nouvelle procédure comme étant en continuité avec la politique du supérieur du gouvernement; le texte 2 se centrant sur la mesure proposée et non la politique qui la porte. Le texte 1 a obtenu 92 voix, le texte 2, 45 voix, et 7 voix, dont celles des deux représentants de la section $\mathrm{CNU} 19$, ont refusé de prendre parti pour l'un ou l'autre texte (sources: liste de diffusion SNESUP, liste de diffusion élus CNU 19). La critique (notamment des « dangers » de la LRU) exprimée par les deux représentants de la section 19, eux-mêmes porteurs d'une motion votée par les membres de la section par 35 voix (dont la mienne) et 1 abstention, visait à refuser l'alternative entre les textes 1 et 2, présentée comme fausse. Le quasi-unanimisme d'un $\mathrm{CNU}$ divisé à l'origine (à proportion de 3/4-1/4) entre deux listes très opposées sur la question des autopromotions de membres du précédent $\mathrm{CNU}$, mais réunifié sur la question de l'évaluation, mérite d'être notée ; classiquement, un collectif de travail peut se ressouder sur un combat commun qui donne du sens à son action et produit de la solidarité professionnelle. La motion regrettait que la discussion sur les différentes modalités de l'évaluation n'ait lieu que dans un cadre global et politique lui-même discutable parce que restreint et orienté; d'où le refus de la discussion sur les modalités. Peut également être critiqué, comme l'autorise l'analyse sociologique la plus basique (au sens de fondamentale), le fait que ce cadre relève d'un « impensé institutionnel » (Bourdieu, 1984, 1988), duquel toute discussion doit partir: à savoir la «conception productiviste du savoir" (motion CNU 19). Pour information, la motion de la section CNU 70 (sciences de l'éducation) est l'une des plus critiques des modalités ministérielles de l'évaluation, mais ne fait ni référence à la LRU ni à un cadre plus global de réification du travail enseignant, seulement à un modèle de l'évaluation plus général, plus décontextualisé et plus abstrait. On constate que, si les collectifs de travail enseignant que constituent les sessions du CNU sont très formalisés, moyennement fréquents et assez intenses, le problème reste le sens des débats qui s'y déroulent.

Pour être tout à fait complet sur cette question du sens, la définition du travail de l'enseignant-chercheur sociologue (et de l'enseignant en sciences économiques et sociales qui peut lui être reliée), telle que la porte la section CNU de sociologie, peut à son tour faire l'objet d'une critique, portant sur son manque d'opposition à la réification du travail enseignant du supérieur et du secondaire. En un mot, il y a selon nous une déqualification et une réification du travail du sociologue si celui-ci ne s'inscrit pas dans une définition claire et un lien direct avec la sociologie générale; et si celui-ci ne définit pas «fermement » les modalités de sa diffusion sociale, de son « utilité ». L'autonomie de la discipline est liée à l'autonomie de travail, l'autonomie de la sociologie à celle du sociologue. Prendre le risque de laisser définir, comme le fait le CNU de sociologie actuel, la sociologie comme une agrégation de spécialités elles-mêmes définies par leur objet plus que par leur démarche, et comme une juxtaposition d'approches théoriques irréductibles et équivalentes alors qu'elles sont identiques (sur certains points), complémentaires et inégalement sociologiques, consiste, toujours selon nous, en une claire déqualification du 
travail de sociologue. Nous ne développerons pas faute de place, mais nous allons maintenant nous appuyer sur une enquête sociologique qui aborde la question de l'autonomie au travail, ou de son absence, et de ses conséquences sur les collectifs de travail, qui fait écho aux remarques qui viennent d'être formulées à travers l'examen des deux contextes.

\subsection{Une enquête de terrain sur le travail collectif des enseignants}

Notre enquête concerne le travail enseignant dans le premier degré. Nous menons depuis 1998 un suivi longitudinal par entretiens semi-directifs sur une cohorte d'une vingtaine d'enseignants volontaires. Ils ont été interrogés à huit reprises, sur les mêmes thèmes, entre $1998(n=26)$ et $2010(n=19$, soit sept « disparus »), soit environ 190 entretiens. Leurs propos ont été recueillis la plupart du temps à l'IUFM, parfois dans les écoles d'affectation, dans un contexte marqué par une certaine proximité, inhabituelle, qui façonne une relation de recherche particulière, comportant une dimension analytique, de retour sur sa propre pratique professionnelle qui, nous semble-t-il, s'est révélée bénéfique pour les données produites. Y compris pour le chercheur, qui a été amené à se poser les questions qu'il posait et à réfléchir aux réponses qu'il pouvait lui-même apporter. Les enseignants de la cohorte représentent un échantillon très homogène : même profession exercée, au même moment, dans un même département, suite à une même formation; âges proches, et toutes des femmes sauf un homme.

L'analyse faite à partir de leurs propos inscrits dans la durée montre que ceux-ci se polarisent assez rapidement dans leur parcours professionnel entre deux logiques, l'adaptation (définie empiriquement, à travers les entretiens, comme recherche, par l'enseignant au travail, de réponses) et l'autonomie (empiriquement: recherche de questions). L'adaptation illustre la « réactivité sans limites » mais aussi « sans action » du travailleur, déjà évoquée (Supiot), et s'inscrit donc dans une logique de réification plus ou moins forte, alors que l'autonomie illustre la « conquête de soi partagée » (Supiot), source d'enrichissement de l'activité (au sens de Clöt, 1997), de critique du «travail abstrait » (au sens de Marx), et de « dé-réification » du travail.

Nous avons montré (Sembel, Léonard, Teruel \& Gesson, 2009) que l'entrée dans la carrière se traduit par un travail d'équipe, quand il existe, dominé par la recherche, via l'adaptation, de « ce qui marche ». Rien ne semble plus étranger à l'enseignant débutant que de passer du temps en collectif qui ne serait pas immédiatement rentabilisé par l'acquisition de nouvelles recettes.

30 Avec le temps, le concept de sociabilité professionnelle (entre collègues), mesuré par la fréquence et l'intensité des contacts professionnels, donne les résultats suivants. Cette sociabilité se répartit en quatre types, selon la fréquence et l'intensité. Dans notre cohorte, deux sont largement dominants, et opposés : 11 cas à forte fréquence et faible intensité (relations au quotidien avec les collègues servant de ressources ponctuelles, passant plus par de la communication informelle que par des réunions); 12 cas à faible fréquence et forte intensité (quelques «collègues-amis » comme personnes ressources régulières, avec lesquelles se forment des "binômes»); deux sont minoritaires, et également opposés : 4 cas à faibles fréquence et intensité (sorte de « repli sur soi »), 2 cas à fortes fréquence et intensité (contacts maximaux). Le total de 29 "possibilités » est supérieur aux 19 professeurs des écoles de l'échantillon, car seuls 9 n'ont connu entre 1998 et 2005 qu'un seul «modèle » de sociabilité professionnelle, 9 en ont connu deux, et 
1, trois. Ces résultats confirment l'existence de deux «modèles» de sociabilité professionnelle collective dominants et classiques. Les deux cas minoritaires permettent de démentir d'une part l'idée d'enseignants massivement repliés sur eux-mêmes (mais aussi, nous le verrons dans le paragraphe suivant, l'idée d'un repli qui serait automatiquement un problème : un enseignant peut s'épanouir sans ses collègues dans son travail) ; et d'autre part l'idée d'enseignants devant être plus présents sur leur lieu principal de travail (l'école ou l'établissement), avec l'idéologie sous-jacente, issue du management des employés de bureau, selon laquelle une meilleure efficacité professionnelle serait corrélée avec une augmentation conséquente de la présence sur le lieu de travail, et dans le cas du travail collectif des enseignants, avec l'augmentation de l'intensité et de la fréquence des relations avec les collègues.

31 Une bonne partie des enseignants en situation d'adaptation possède une sociabilité assez «forte», avec des liens fréquents avec les collègues, mais souvent avec une faible intensité, de manière ponctuelle et au sein du lieu d'enseignement. Le travail d'équipe qui en découle sert ici à favoriser l'adaptation:

Il y a une relation entre adultes qui fait qu'on essaie d'avoir un discours toujours pareil et par rapport aux familles. Bon après, travail d'équipe réellement, c'est pas toujours possible. On le fait lors des réunions mais après y'a aussi du relationnel de personnes. (Fabienne M.)

La communication entre enseignants est alors perçue comme fondamentale : «Et ça change la vie parce qu'on s'entend très bien donc il y a aussi du relationnel. On s'entend très bien, on communique très bien » (Carole B.). La dimension professionnelle du travail en équipe peut également être mise à l'écart au profit de l'aspect relationnel et de ses " vertus adaptatives », assurant la cohésion et évitant les conflits entre collègues :

Nous on est six filles, sept avec la décharge de direction, et on est toutes à peu près sur la même longueur d'onde. Y'en a pas une qui choque ou qui dénote. Si c'était le cas ce serait difficile quand même... » (Fabienne B.)

Une autre partie se distingue par sa sociabilité plus «faible», avec des contacts professionnels peu fréquents. Combinés avec une faible intensité, ils constituent une adaptation par repli sur soi qui peut se traduire par un rejet du travail d'équipe et de la relation aux collègues :

J'ai rien contre mais moi dès que je suis en équipe je fais rien. C'est comme ça, je me laisse porter. Et je fais même en équipe des choses qui ne m'intéressent pas parce que c'est en équipe, je ne veux froisser personne, alors ça me plaît pas et je fais quand même. Mais au final ça me convient pas. Moi je trouve que c'est beaucoup de perte de temps. (Caroline V.)

Si j'ai personne avec qui travailler l'an prochain, je prendrai des manuels, mais je referai pas tout à fond, parce que c'est prendre beaucoup de risques quand même

(...) Le degré d'autonomie, il est tellement grand que certains n'en font rien (...)

C'est vrai que la fiche à remplir, c'est extrêmement sécurisant. (Fabienne M.)

Mais cette faible fréquence peut s'articuler aussi avec une forte intensité relationnelle, et déboucher sur une adaptation par division du travail avec un collègue, ce qui est souvent le cas lors de la prise de poste, pour surmonter l'urgence. Faire appel à un collègue rend dans ce cas la somme de travail moins lourde. Certains enseignants perpétuent cette façon de faire même lorsqu'ils ne se trouvent plus dans cette période délicate. Ils sortent de l'adaptation forcée du début et passent à une adaptation choisie. Souvent, la personne avec laquelle ils vont travailler ne se trouve pas dans la même école, ce qui permet aussi de contourner certains désagréments du travail en équipe : «On travaille à deux et c'est plus facile que de travailler en équipe. Parce que c'est difficile par rapport au caractère de 
chacun et surtout aux emplois du temps » (Christelle C.). Cette division du travail avec un collègue permet toujours de faire face efficacement au travail demandé :

Et puis maintenant on se connait bien donc on se conteste de moins en moins parce qu'on se comprend de mieux en mieux donc c'est une économie de temps énorme. Ça se fait presque tout seul maintenant, donc on se complète, c'est formidable, on fait pas les mêmes choses et comme on n'aime pas les mêmes choses...(...) J'ai renoncé à vouloir être trop ambitieuse. » (Isabelle G.)

Le travail collectif informel des enseignants adaptés correspond à ce que nous avons appelé l'« altruisme restreint", caractérisé notamment par du «bricolage » et de la réflexivité «faible », l'objectif du collectif étant l'efficacité (c'est-à-dire l'immédiateté) des résultats obtenus par la coopération, ce que Durkheim critiquerait comme étant un échange trop spécialisé et Mauss comme un échange trop intéressé (Sembel \& Gesson, 2011). L'enjeu est ici la maitrise du métier et non l'accomplissement du travail (cf. aussi Sembel, 2013b), dont la déqualification, la réification et la désintellectualisation ne posent pas de problèmes insurmontables, si tant est qu'ils soient identifiés comme des problèmes par les travailleurs concernés.

Une partie des enseignants en situation d'autonomie connaît une sociabilité professionnelle à relations fréquentes et intenses :

Ça me plait à cause du travail d'équipe, de l'esprit d'équipe. On sait qu'on peut s'appuyer les uns sur les autres et les enfants aussi le savent. Ce qui fait que tous les problèmes que l'on peut rencontrer à l'école sont diminués à mon sens grâce à la cohésion d'équipe et à un discours cohérent de l'équipe. (Isabelle $\mathrm{H}$.)

"J'en discute beaucoup avec tous les enseignants que je croise, c'est aussi de la formation.» (Gwendoline G.). L'aspect relationnel du travail d'équipe est également valorisé : «Après on a ses petites habitudes. Y'a un besoin de renouveler, de faire autre chose, d'échanger avec d'autres personnes.»(Christine V.L.). Cette sociabilité peut également se développer en dehors du lieu d'enseignement: «Après le groupe Freinet c'est pas un engagement associatif, mais c'est vraiment un endroit où ça m'a fait du bien d'aller pour la motivation aussi» (Gwendoline G.). Enfin, cette sociabilité forte permet aux enseignants de faire face à la solitude qui peut parfois les guetter: "On essaie de travailler en équipe ici, de faire des projets un peu communs, ça permet de ne pas travailler seul » (Nathalie L.). Car ils peuvent aussi construire, plus que les adaptés, leur autonomie par le repli sur soi (faibles intensité et fréquence des contacts), ne serait-ce que par les exigences imposées à soi qui ne se partagent pas toujours, ou par les ruptures nécessitées par l'exigence d'autonomie : «J'expérimente beaucoup de choses (...) Me dire que ça ne va pas sans arrêt, c'est un moteur pour faire mieux. Mais c'est un moteur qui est bouffant à la longue parce qu'on n'est jamais satisfait de ce que l'on fait» (Nathalie C.) ; "Si j'avais plus de temps je pourrais faire des choses mieux» (Nathalie L.). Plus globalement, ce travail collectif correspond à un altruisme "élargi », construit sur la durée (éventuelle), sans recherche d'un bénéfice immédiat ou visible, dans un processus de diffèrement pleinement «maussien » du « retour » du don (Sembel \& Gesson, 2011). Selon nos résultats, cet altruisme n'est pas absent chez les enseignants débutants, mais il est plus fréquent chez ceux qui ont connu l'expérience de la mobilité et de la stabilité; et il permet de garder une distance certaine avec les routines et la sociabilité professionnelle trop fréquente et peu intense (ce qui peut être retenu comme une définition de la routine). La distance au métier permet ici l'investissement dans le travail « réel », une certaine lutte contre la réification et pour l'intellectualisation. 
38 La dynamique de l'altruisme restreint recherche plutôt un équilibre par la force « contrôlée » des liens formels, celle de l'altruisme élargi cherche à se développer par la force « continue » des liens faibles. Dans le premier cas, l'équilibre, une fois atteint, doit être préservé ; dans le second, la recherche de sens (et non d'équilibre) semble cultiver une dynamique collective sans fin (cf. Sembel et al., 2012). Mais cette différence, lourde de conséquences sur le travail individuel, ne doit pas masquer un rapprochement du travail collectif adapté ou autonome, du point de vue des formes de sociabilité professionnelle qu'il finit par prendre. La différence entre eux se situe ailleurs, au niveau du sens imprimé par les logiques d'adaptation et d'autonomie sur le contenu du travail, et ce, quelle que soit la forme qu'il prend.

\section{Discussion des résultats}

Le principal résultat tiré du premier contexte (la mastérisation) est le suivant: les travailleurs les plus au cœur d'un processus de transformation de leur travail ne sont pas forcément les mieux placés pour penser ce qui le transforme, et défendre leurs intérêts. Mais, lorsque ces travailleurs sont des enseignants, parmi lesquels nombre d'universitaires, l'argument de Trentin trouve un développement insoupçonné : ce n'est plus seulement la "gauche", mais les intellectuels, qui sont dans l'incapacité de penser leur travail, le travail, sous l'angle d'une valorisation dynamique. Au contraire, ils peuvent accompagner involontairement sa dévalorisation par déqualification, réification et, comble du paradoxe, déintellectualisation. Ni le recours généralisé à la notion de compétence, ni l'expertise pédagogique ou formative, ne freinent cette dévalorisation et ne nourrissent une réflexion et une action qui s'y opposeraient.

Le principal résultat tiré du second contexte (le CNU 19) est le suivant: quand, exceptionnellement, suite à un contexte interne très particulier (les " autopromotions »), un collectif de travail d'enseignants-chercheurs résiste à ce qu'il identifie comme un avatar de cette dévalorisation (la question de l'évaluation), il s'isole des autres collectifs placés dans la même situation (les autres sections $\mathrm{CNU}$ ), qui ne voient pas la dévalorisation, et se heurte bien sûr à l'opposition de la tutelle, face à laquelle il se trouve aujourd'hui en situation de quasi-désobéissance civique (avec son refus, toujours dans la même logique, d'évaluer les demandes de primes doctorales). En somme, le positionnement marginal et en porte-à-faux du CNU 19 est une exception qui confirme l'argumentation de Trentin.

41 Le principal résultat tiré de l'enquête de terrain (les professeurs des écoles) est le suivant: quelles que soient les formes que peut prendre le travail enseignant collectif, selon sa fréquence et son intensité, l'essentiel se situe ailleurs que dans la formalisation retenue. Le problème n'est pas d'inciter les enseignants à travailler ensemble, mais de savoir quel rapport ils entretiennent individuellement avec leur travail, pour savoir quel sens leur travail collectif aura. Celui-ci peut être le travail collectif superficiel des enseignants adaptés qui restent centrés sur eux-mêmes : la solidarité est absente, le collectif est une agrégation ponctuelle et limitée le temps du rassemblement, le travail collectif ne donne lieu qu'à des échanges immédiatement intéressés, dans le cadre d'un «altruisme restreint». Le sens du travail collectif des enseignants autonomes est tout autre : sortant d'eux-mêmes, au sens de Durkheim et de Mauss, ils sont collectifs par définition, solidaires de fait, et ne travaillent pas ensemble en fonction d'intérêts 
ponctuels. Et lorsque ceux-ci sont présents, ils alimentent le processus général de « dette solidaire ». Leur altruisme est « élargi ».

Ces constats, critiques et sociologiques, laissent transparaître des pistes d'action. Quelles sont les perspectives offertes à la sociologie pour remplir son rôle d'utilité sociale ? En un sens, le collectif est partout dans le travail enseignant. Les politiques publiques actuelles en valorisent une forme bien précise, celle de l'altruisme restreint. La question sociologique est donc moins de définir le travail collectif, car toute définition réduirait sa diversité empirique ; ni de le réguler normativement, car toute régulation de ce type (les plus répandues et souvent les seules proposées) reviendrait à le contrôler et donc à réduire, à nouveau, sa diversité. L'objectif sociologique d'analyse et d'action se situe non pas au niveau de la forme idéale de collectif à atteindre, telle une norme idéologiquement imposée, mais au niveau du sens à donner à ces collectifs. De la manière de les intégrer autour d'un idéal dynamique pour que la régulation des pratiques professionnelles se mette en place dans les meilleures conditions, si l'on reprend le vocabulaire durkheimien. Le rôle du chercheur s'élargit : à partir de sa posture impliquée et décalée, il doit dégager le sens du travail collectif pour que le collectif s'en empare. Le collectif n'est pas à créer formellement, il est à identifier comme dynamique.

Les enseignants de la «base » sont aussi démunis pour théoriser leur travail collectif que les enseignants du «sommet » qui les forment ou les représentent. Ce qui relativise la différence de position entre les deux, d'où la mobilisation de ces catégories entre guillemets, en réalité purs artefacts. Mais les premiers le vivent au quotidien, et la recherche (parfois dans un contexte de recherche-action, ou de travail "personnel») peut leur permettre de construire une distance réflexive structurée voire didactisée, ellemême étape vers (dans le domaine sociologique qui est le nôtre) une socioanalyse (au sens de Bourdieu, 2004), et un travail collectif plus développé (au sens de « développement professionnel » chez van Zanten, 2001; ou "développement de l'activité » chez Clôt, 1997). Le collectif peut être puissant, son "développement professionnel » pourrait transformer de façon insoupçonnée les pratiques de travail, et donc enrichir ce que Marx appelait la force de travail, principale ennemie de la réification et de la déqualification de ce dernier.

Il est possible de faire l'hypothèse que ce qui manque n'est pas la dimension collective du travail enseignant, mais, pour développer cette dimension toujours forte potentiellement, un niveau de régulation intermédiaire (Maroy, 2006) entre, d'une part, le ministère et les think tank qui alimentent ses réflexions, et d'autre part les écoles et les établissements, pour tenter de lui donner un sens. Ce niveau «méso » de régulation n'est pas suffisant en soi, tant les processus de bureaucratisation d'une part (Hibou, 2012), de reproduction et de production des inégalités d'autre part, sont puissants, en France comme en Europe, du côté du système scolaire comme des parents d'élèves. L'entrée et l'implication (variable socialement) de ces derniers dans les écoles et les établissements ne garantit aucunement l'amélioration de l'ensemble, et se traduit souvent par un contrôle supplémentaire du travail enseignant loin d'être toujours pertinent (Maroy, 2006). Il permettrait toutefois d'opposer quelque chose de formalisé (et différent de ce qui existe déjà au niveau méso) au top-down "macro", centralisateur, encore défendu, et utilisé à plein, par nombre d'experts de la dérégulation et du New public management; et au bottom-up «micro", hyper-localisé, source, sous couvert d'autonomie, de toutes les dérives en termes de néoféodalisme (sur lequel un courant de pensée décentralisateur attire pourtant l'attention). Dans les deux cas, le travail collectif enseignant est pris en tenaille entre un « haut » et un 
«bas » qui renforcent en son sein les tendances, déjà repérées par Marx, au « despotisme de fabrique » et à l'« idiotisme du métier » (autres caractéristiques de la réification du travail, y compris enseignant). Le Café pédagogique ne remarquait-il pas, lors de la rentrée de septembre 2013, que les réformes du métier d'enseignant semblaient vouloir transformer ce dernier en "employé devant rendre des comptes»? Le recours au "méso " garantirait des échanges élargis échappant à l'immédiateté, une distance critique aux routines sans réflexion (Durkheim, 1938/1990), une véritable autonomie professionnelle (autour de la recherche de questions plus que de réponses, ainsi que nous l'avons définie).

Ces dernières considérations renvoient au problème sociologique selon nous le plus fondamental dans la question du travail des enseignants, de leur formation, de leur identité professionnelle, de sa dimension collective : la critique d'une culture de groupe social, de classe favorisée, laquelle constitue le plus petit dénominateur commun à tous les statuts, toutes les disciplines, tous les savoirs, tous les niveaux d'enseignement, toutes les inégalités (sociales et de genre), tous les publics, et finalement tous les rapports que chaque enseignant, lui-même membre des catégories favorisées (de par son diplôme, et/ ou son statut), entretient individuellement et collectivement avec son travail. Le "pire " collectif de travail identifié par les recherches étant le conseil de classe. L'agrégation des cultures de classe individuelles forme à cette occasion un collectif produisant des inégalités sociales supplémentaires (Duru-Bellat, 2002). La critique de telles pratiques collectives ne peut être que sociologique. L'occasion d'une socioanalyse collective, dont les modalités restent tout entières à préciser, si le principe était retenu, pourrait être saisie. La véritable « libération » de la parole qui se produit lors de moments collectifs tels que certains stages de formation continue, de mobilisation politique, de rencontres privées « où l'on ne va pas passer la soirée à parler boulot », et pourtant si, montre que le terreau est fertile pour une telle socioanalyse collective.

\section{Conclusion}

Les conséquences politiques de la validation sociologique de l'existence théorique et empirique de la dimension collective du travail enseignant, et des invariants structurels historiques auxquels elle renvoie, ne sont pas négligeables.

La politisation «par le haut » du travail enseignant, par la mastérisation et l'évaluation; et "par le bas" par l'existence, valorisée, de l'adaptation, parachève un processus historique (au sens de diachronique) de rationalisation technique du travail, qui touche le travail des enseignants, fait débat depuis les origines de la mise en place de leur formation "professionnelle», et semble s'accélérer aujourd'hui, malgré les multiples résistances sur le «terrain» (écoles, établissements du secondaire et du supérieur, organismes de formation) que cette évolution suscite parfois.

Un tel processus ne supprime pas les collectifs de travail, il neutralise la capacité de la plupart des acteurs (syndiqués ou non, individuels ou collectifs) et des chercheurs, à identifier et à critiquer la réification du travail qui l'accompagne.

Dire qu'un tel travail n'existe pas, le déplorer et vouloir l'organiser revient à déréguler l'existant, surtout informel, au lieu, par exemple, de le reconnaître, voire de le renforcer ; c'est clairement ce qui nous semble à l'œuvre par le truchement de tout ce qui tourne autour de la compétence des enseignants comme des élèves, c'est-à-dire des effets sociaux 
de l'utilisation de la notion de compétence; ainsi que le recours à la « redéfinition des missions» des enseignants (Thélot, 2004), presque toujours dans le sens de la déqualification de leur travail, sous couvert de modernisation, de réforme, etc.

Face à cette offensive plus idéologique que scientifique, ou encore idéologique sous couvert de scientificité, qui vise à définir le travail enseignant comme un collectif d'employés de bureau (caractérisé par une présence accrue, des missions élargies, un affaiblissement du rapport à la discipline ou à la polyvalence, des collectifs trop localisés, une évaluation "adaptative»), comme un travail «abstrait» (Marx), la faiblesse des réflexions et actions collectives, pour ne serait-ce que reconnaître les aspects de cette réification, est assez frappante.

Affirmer au contraire que la dimension collective est intrinsèque au travail enseignant, qu'il est par exemple aussi difficile pour un enseignant de travailler tout le temps seul qu'à plusieurs (c'est-à-dire au moins avec un collègue), permet de renverser le problème, de fonder une véritable construction d'objet, et d'intégrer cette construction dans les aspects les plus larges des questionnements sur le travail, en participant par exemple de la (re)définition du concept d'autonomie dans le travail. Les conditions politiques, pédagogiques et didactiques de la reconnaissance et de la valorisation d'une autonomie ainsi redéfinie deviennent un enjeu plus décisif que jamais. Finalement, l'objet sociologique " travail collectif enseignant », marginal, peu visible voire dénié, pourrait en réalité être doté d'une dimension heuristique inattendue pour nourrir les réflexions sur les questions du travail en général.

\section{BIBLIOGRAPHIE}

Barrère, A. (2002). Les enseignants au travail. Paris : L'Harmattan.

Bourdieu, P. (1984). Homo academicus. Paris : Minuit.

Bourdieu, P. (1988). L'ontologie politique de Martin Heidegger. Paris : Minuit.

Bourdieu, P. (2004). Esquisse pour une auto-socioanalyse. Paris : Raisons d'agir / Le Seuil.

Clôt, Y. (1997). Le travail sans l'homme? Paris : La Découverte.

Dobry, M. (1986). Sociologie des crises politiques. Paris : PFNSP.

Dubet, F. (1991). Les lycéens. Paris : Le Seuil.

Dubet, F., \& Martuccelli D. (1996). À l'école. Paris : Le Seuil.

Duru-Bellat, M. (2002). les inégalités sociales à l'école. Paris : PUF.

Durkheim, É. (1893/1986). De la division du travail social. Paris : PUF.

Durkheim, É. (1938/1990). L'évolution pédagogique en France. Paris : PUF.

Geay, B. (2010). Les néo-enseignants face à l'utilitarisme. Actes de la recherche en sciences sociales, 184, 72-89.

Granovetter, M. (1973/2008). Sociologie économique. Paris : Desclée de Brouwer. 
Hibou, B. (2012). La bureaucratisation du monde à l'ère néo-libérale. Paris : La Découverte.

Malet, R. (2008). La formation des enseignants comparée. Berne : P. Lang.

Marcel, J.-F. (2006). Le collectif d'enseignants. Explorations théoriques et empiriques d'un nouvel acteur des systèmes éducatifs. Formation et pratiques d'enseignement en question, 5, 85-99.

Maroy, C. (Éd.). (2006). École, régulation et marché. Paris : PUF.

Mauss, M. (1950). Sociologie et anthropologie. Paris : PUF.

Normand, R. (2007). L'Europe de l'éducation : entre management et politique. Lyon : INRP.

Sembel, N. (2003). Le travail scolaire. Paris : Nathan.

Sembel, N. (2013a). La définition du « bon » fonctionnaire comme enjeu de luttes. In J.-F.

Dupeyron \& C. Miqueu (Éds.), Éthique et déontologie dans l'Éducation Nationale (pp. 43-55). Paris : A. Colin.

Sembel N. (2013b). Le travail enseignant au primaire : l'adaptation comme passage, l'autonomie comme rupture. In P. Cordazzo \& B. Fichet (Éds.), Transition, passage en sciences sociales (pp. 41-55). Strasbourg : Néothèque Éditions.

Sembel N., Gesson B., Léonard F. \& Teruel B. (2012). La professionalisation des enseignants en IUFM entre idéal et critique : de l'utilitarisme à la satisfaction intellectuelle. In E. Quenson \& S. Coursaget (Éds.), La professionnalisation de l'enseignement supérieur. De la volonté politique aux formes concrètes (pp. 133-146). Toulouse : Octarès, 2012.

Sembel, N., \& Gesson, B. (2011). Deux logiques de la spécialisation du travail « altruiste » des professeurs des écoles : adaptation « intégrée » ou autonomie « distanciée » ? In L. Jacquot \& C. Kraemer, Travail et dons (pp. 251-266), Nancy : PUN.

Sembel N., Léonard F., Teruel B. \& Gesson B. (2009). L'entrée dans la carrière d'une cohorte de Professeursdes écoles en Gironde : la construction de l'identité au travail entre adaptation et autonomie. In R. Goigoux, L. Ria \& M.-C. Toczek-Capelle (Éds.), Les parcours de formation des enseignants débutants (pp. 135-147). Clermont-Ferrand : Presses Universitaires Blaise Pascal.

Supiot, A. (2012). Introduction. In B. Trentin, La cité du travail, le fordisme et la gauche (pp. 13-31). Paris : Fayard.

Thélot, C. (2004). Pour la réussite de tous les élèves. Paris : La documentation française.

Trentin, B. (2012/1997). La cité du travail, le fordisme et la gauche. Paris : Fayard.

Woods, P. (1990). L'ethnographie de l'école. Paris : A. Colin.

Van Zanten, A., \& Grospiron, M.-F. (2001). Les carrières enseignantes en ZEP. Ville-ÉcoleIntégration, 124, 224-268.

\section{RÉSUMÉS}

Notre approche du travail collectif des enseignants est résolument sociologique. En partant à la fois du travail informel inscrit dans la sociologie interactionniste et du travail réifié relevant de la sociologie marxiste et de certains de ses développements actuels, combinant donc points de vue "micro-empirique» et "macro-historique», nous montrerons en quoi des contextes professionnels traversés par des logiques contradictoires de déqualification et de réintellectualisation du travail enseignant, s'éclairent par l'analyse sociologique du rapport au travail des enseignants. Notre principal résultat est la difficulté de ces derniers à formaliser 
individuellement et collectivement la déqualification et la réification de leur travail; la principale voie de sortie d'une telle impasse est une double action aux niveaux "mésoorganisationnel » et "socioanalytique collectif », qui restent largement à mettre en œuvre, et constituent selon nous les conditions pour une autonomie professionnelle condition d'un véritable travail collectif

Our approach of teachers' collective work is decidedly sociological. Starting from both the informal work of the interactionnist sociology and reified work of marxist sociology and certain of its current developments, thus combining perspectives "micro-empirical" and "macrohistory", we will show how some professional contexts, through with conflicting logics of deskilling et re-intellectualization of teacher's work, can be enlightened by the sociological analysis of this work. Our main result is the difficulty of teachers to formalize, individually and collectively, the deskilling and the reification of their work; the main way out of this cul-de-sac is a double action towards "meso-organizational" and "socioanalytical collective" levels, which remain largely to implement, and are, according to us, the conditions for a professional autonomy, which is necessary for the collective work.

\section{INDEX}

Keywords : sociology, work, teachers, deskilling, autonomy

Mots-clés : sociologie, travail, enseignants, déqualification, autonomie

\section{AUTEUR}

\section{NICOLAS SEMBEL}

Centre Émile Durkheim, Bordeaux et ESPÉ d'Aquitaine 